\title{
Elbow joint rehabilitation equipment actuated by pneumatic muscles
}

\author{
Georgiana Vetrice $^{1, *}$, and Andrea Deaconescu ${ }^{1}$ \\ ${ }^{1}$ Transilvania University of Braşov, Department of Industrial Engineering and Management, 500036 \\ Braşov, 29 Eroilor Bd., Romania
}

\begin{abstract}
The mobility of the limbs is an essential prerequisite for the individual's physical autonomy. For persons suffering from post-traumatic affections of the elbow joint such limited mobility results in barriers in fulfilling personal or professional tasks. Passive motion has certain specific advantages and beneficial effects, thus being highly recommended for the recovery of injured joints. The paper presents a model of rehabilitation equipment that induces continuous passive motion of the elbow, as part of a recovery programme. The equipment is actuated by pneumatic muscles, using compressed air as the source of energy that generates force and motion. The main benefits of the pneumatic actuation system compared to other driving systems are its low cost, simple and robust construction and swift response to commands. Its constructive simplicity and reduced cost adds to the system's eligibility for deployment in medical units.
\end{abstract}

\section{Introduction}

The movements of the upper limbs are essential for the individual. Lack of mobility can have a major impact on social life. In the case of patients suffering from post-traumatic affections of the elbow joint (Fig. 1.), natural recovery of mobility can take up to several months. In order to ensure swift rehabilitation continuous passive motion is recommended, a postoperative treatment protocol which consists in applying a range of slow, rhythmical and continuous movements to the joint, without contracting the patient's muscles. In order to obtain fast results, this procedure can be used from the next day after surgery.

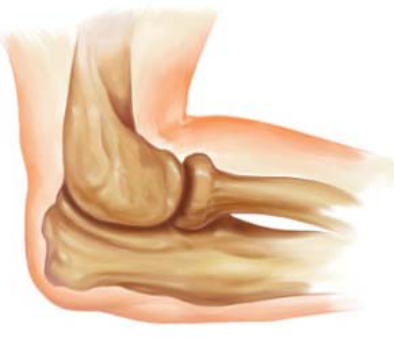

Fig. 1. The elbow joint.

\footnotetext{
*Corresponding author: vetrice.georgiana@unitbv.ro
} 
The elbow joint is responsible for two movements: flexion-extension and pronationsupination. Flexion is obtained by moving the forearm close to the arm, and extension by moving it away from the arm. Pronation is the rotation of the forearm by its longitudinal axis, so that the thumb is turned towards the body, while supination is the rotation of the forearm by its longitudinal axis, when thumb is turned away from the body. In the wake of surgery, the individual's capacity of conducting these movements may be limited, thus calling for specially designed equipment for the speedy rehabilitation of the elbow joint.

\section{Elbow rehabilitation equipment}

Using rehabilitation equipment considerably reduces the recovery period and the quantity of drugs consumed. Thus, the total costs entailed by recovery are diminished [1]. Most rehabilitation equipment which is nowadays available on the market is driven by electric motors and has a rigid structure. Several such examples are:

- E3 Elbow CPM Device, (Fig. 2a), is a product of OttoBock company, which allows both flexion-extension and pronation-supination [2];

- Fisiotek LT-G Device, (Fig. 2b) also allows both movements, flexion-extension and pronation-supination [3];

- Kinetec 6080 Elbow CPM Device, (Fig. 2c), performs two types of movements: flexionextension or flexion-extension with synchronized pronation-supination [4].

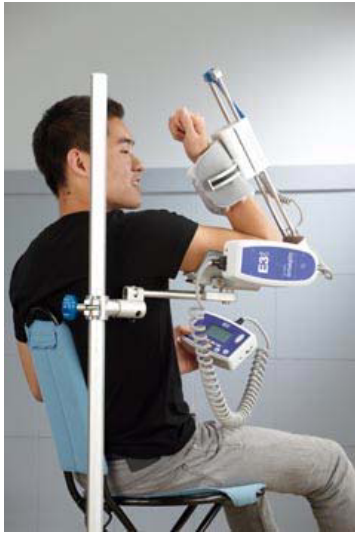

a. E3 Elbow CPM Device
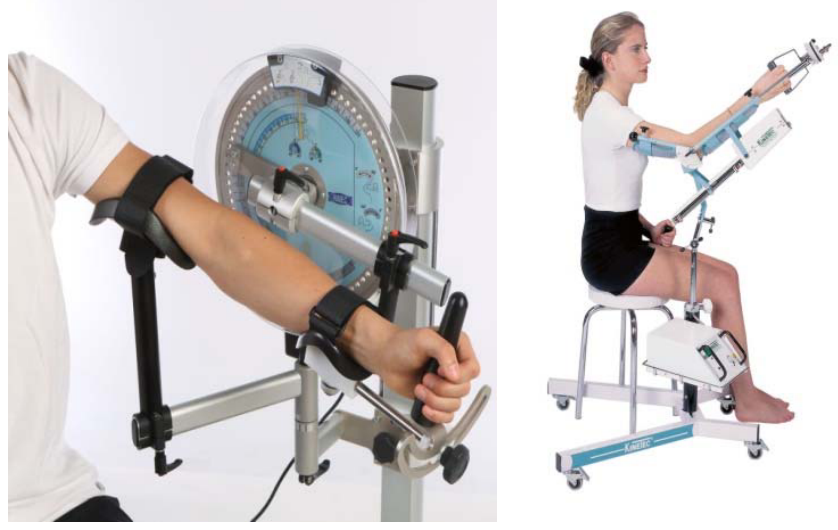

b. Fisiotek LT-G Device

c. Kinetec 6080 Elbow CPM Device

Fig. 2. Electrically driven devices.

Another category is that of rehabilitation equipment endowed with pneumatic actuation systems, characterized by the low weight of the components, favourable response to commands, compliance due to air compressibility, and low maintenance. The following pneumatic muscle actuated rehabilitation devices were patented.
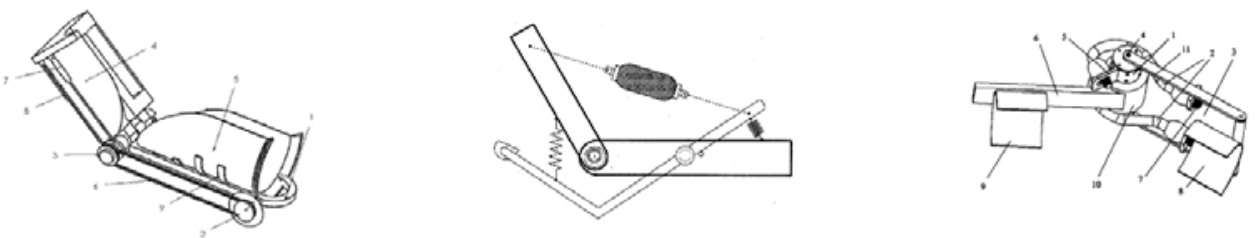

Fig. 3. Pneumatically actuated devices. 
Chinese Xi'an Jiaotong University developed a rehabilitation equipment (Fig. 3a) which, by contracting the pneumatic muscle causes the movement of the forearm and thus, of the joint [5].

Jiliang University of China developed a pneumatic muscle actuated joint (Fig. 3b). The pneumatic muscle contraction/relaxation generates the rotation of the left bar around the joint [6].

The patent granted to Zhejiang University of China refers to a device actuated by two pneumatic muscles (Fig. 3c), which rotates the elbow joint and the upper-arm by controlling the air pressure [7].

This paper presents a constructive solution for elbow rehabilitation equipment using continuous passive motion. The absolute novelty consists in its actuation (by means of pneumatic muscles) and in making the joint movement possible by two axes.

\section{Working principle of the pneumatic muscles}

The pneumatic muscle is an elastic element which, under air pressure, enlarges its diameter and reduces its length. Thus, it carries out a stroke directly proportional with the feed pressure. Fig. 4 first shows the muscle in relaxed state, for zero pressure, and then the contracted muscle for a positive value of the pressure.
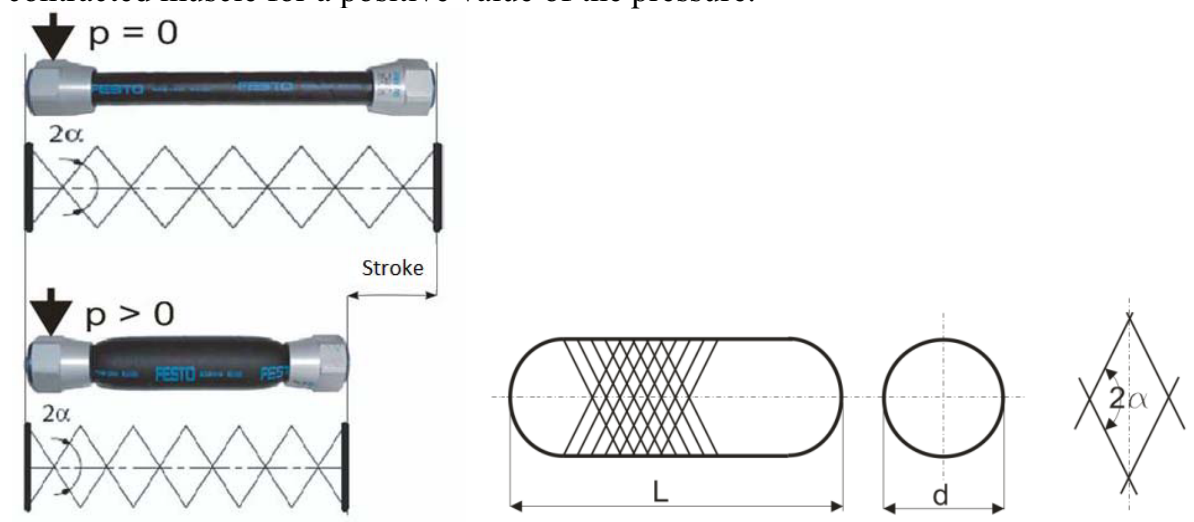

Fig. 4. Working principle of the pneumatic muscle.

An artificial pneumatic muscle includes an interior tube made from an elastic material, usually neoprene. The tube is wrapped in a multilayer tissue made from nylon, in order to give it strength and to protect it from the influences of the working environment. Figure 4 also presents the geometry of the tissue that the interior tube is wrapped in. It can be observed that, in order to perform a stroke, the behaviour of the tissue is similar to the one of a pair of scissors. The behaviour of the pneumatic muscle is similar to that of a spring, meaning that at the end of the stroke the developed force is zero.

If one muscle is not able to achieve the desired movements, various solutions for series or parallel mounting of several muscles can be found.

The main advantages of pneumatic muscles are low price, high power to weight ratio, the possibility of deployment in wet environments, silent operation etc. [8].

In the case of the rehabilitation equipment proposed in this study two pairs of muscles were used, one for each movement performed by the elbow joint. Fig. 5 illustrates the operation of the muscle causing pronation-supination. It can be noticed that the rotation of the joint by means of pneumatic muscles is achieved similarly to the operation of human muscles, based on the agonist-antagonist principle. While a muscle is contracted, the other one is relaxed. 


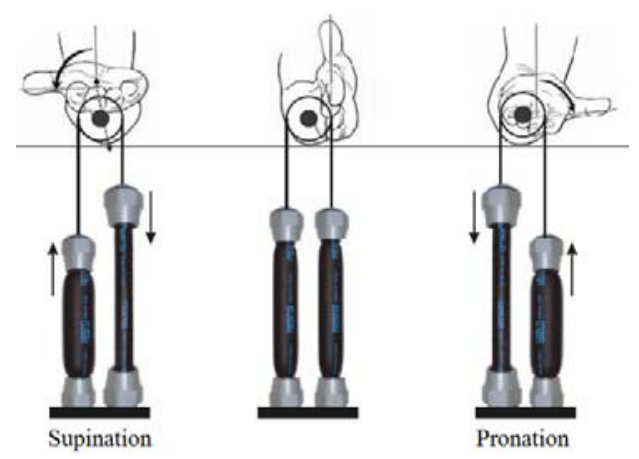

Fig. 5. The operation of the muscle causing pronation-supination.

The same principle is used in the case of flexion-extension (Fig. 6). At zero position, the feed pressure in both muscles is equal; for the movement to take place one muscle is fed compressed air, while the other one relaxes.

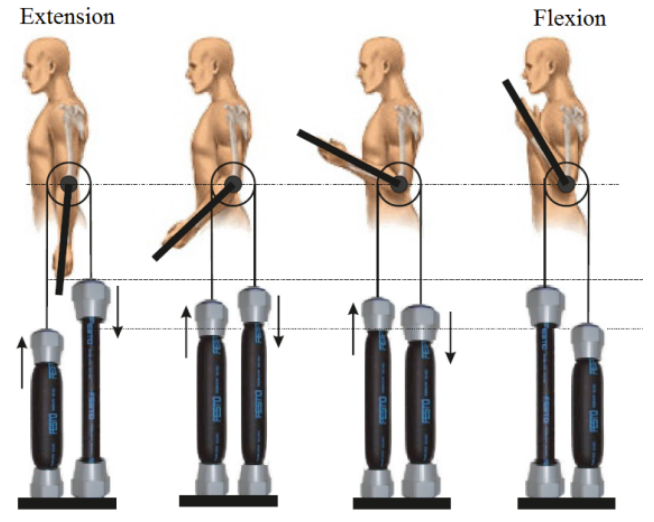

Fig. 6. The operation of the muscle causing flexion-extension.

\section{Proposed rehabilitation equipment}

Fig. 7 shows the kinematic diagram of the proposed equipment, and also the positioning the pneumatic muscles in relation to the human arm in order to perform the movements necessary for recovery. The first pair of muscles causes flexion-extension, while the second one, placed perpendicularly to the first pair, causes pronation-supination movement. These movements are conducted one at a time, separately and not simultaneously. For the correct deployment of the equipment, the patient needs to be in a comfortable position.

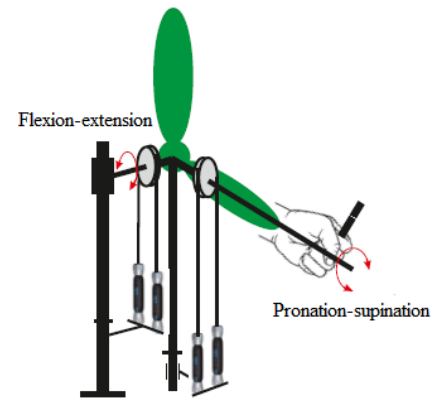

Fig. 7. Position of the patient upper limb in the equipment. 
The geometrical model of the elbow rehabilitation equipment (Fig. 8) was developed based on the proposed kinematic diagram.

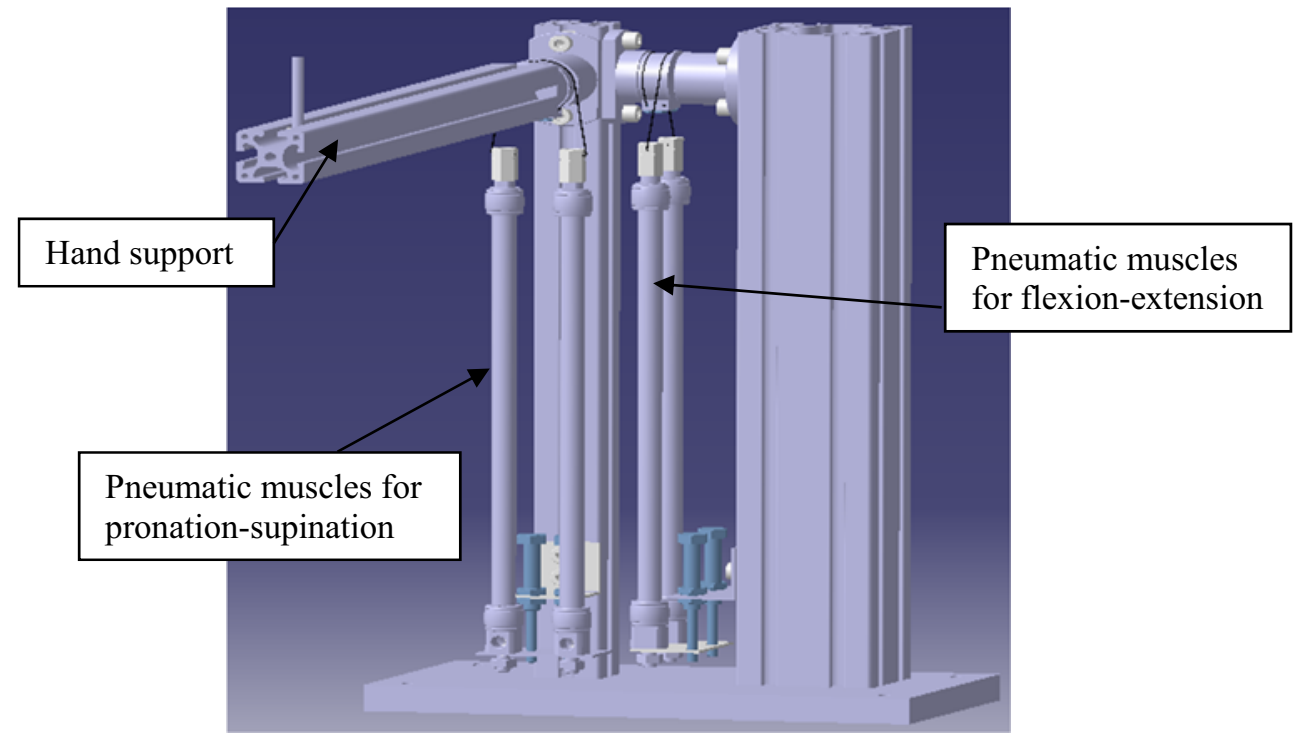

Fig. 8. Construction of the rehabilitation equipment.

The movements made by the hand support are the elbow movements that need to be recovered. For flexion-extension, the rotation of the hand support is generated by using a pair of pneumatic muscles, working in agonist-antagonist mode. For pronation-supination, the movement of the hand support is obtained by rotating it by its longitudinal axis, by means of the second pair of muscles, also working in agonist-antagonist mode.

Fig. 9 presents the construction of the two rotation modules of the rehabilitation equipment.

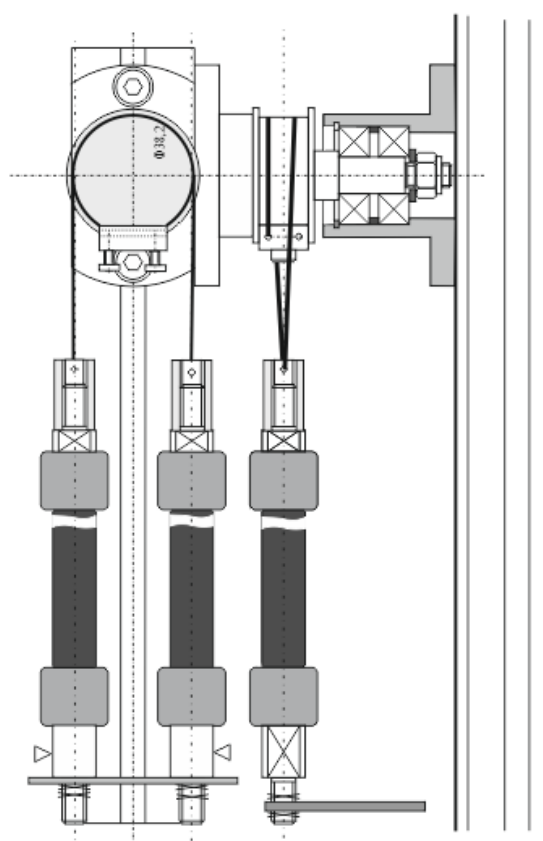

Fig. 9. Rotation modules of the rehabilitation equipment. 
As can be noticed, the free ends of the pneumatic muscles are connected by a wire, drawn over a pulley and fixed in a specially designed structure. Thus, by feeding of compressed air, the movement of the pair muscles one against the other causes the rotation of the shaft by its axis and also the movement of the hand support. The working principle is suitable for both flexion-extension and pronation-supination.

The two pairs of pneumatic muscles used in the construction of the equipment are of 10 $\mathrm{mm}$ interior diameter and initial length of $300 \mathrm{~mm}$. The maximum possible stroke of the free end of the muscle is approximately $20 \%$ of its length in relaxed state (that is $60 \mathrm{~mm}$ ).

\section{Conclusion}

Regaining the mobility of the limbs is very important in the case of patients with posttraumatic disorders, such as to enable them to satisfy their needs and achieve their goals. The deployment of rehabilitation equipment aimed at the recovery of the functions responsible for movement is bound to considerably improve patient personal and professional life. Rehabilitation equipment based on continuous passive motion has the advantage of being usable in both medical units and in patients' homes. This is important for them because in this way they have the chance to use it in the comfort of their own homes. It is well-known that most of the devices developed so far for the rehabilitation of patients with post-traumatic disabilities are electrically driven and thus lacking userfriendliness.

This paper presents and discusses the mechanical structure and the actuating system of a piece of innovative elbow rehabilitation equipment for patients with post-traumatic afflictions. The purpose of designing such equipment was explained together with its concept.

In summary, the pneumatic actuating system endows the equipment the advantages of compliance, low cost and operational simplicity. Therefore, the authors feel the pneumatic equipment is eligible to replace the electrical devices. Further on, this new type of equipment will be computer simulated, built and tested, in order to verify the compliant behaviour of these actuators.

\section{References}

1. A. Deaconescu, T. Deaconescu, IMECS, 2, 1823 (2009)

2. Otto Bock, http://www.ottobock.com/ Accessed: 20.04.2016

3. Rimec, http://www.rimec.it/ Accessed: 20.04.2016

4. Kinetec, http://kinetec.fr/en/ Accessed: 20.04.2016

5. J. Liu, Z. Yifang, S. Yingshuang, Z. Yongqiang, Z. Wei; Y. Qingtai, Elbow medical rehabilitation device based on pneumatic artificial muscle drive. CN203263743 (2013)

6. Y. Dongming, W. Binrui, J. Yinglian, Pneumatic muscle flexible elbow joint device with buffer spring and flexible shaft sleeve. CN202071080 (2011)

7. Y. Canjun, Z. Jiafan, Z. Jie, C. Ying, Flexible exoskeleton elbow joint based on pneumatic power. CN200984250 (2007)

8. A. Deaconescu, T. Deaconescu, WCE, 2, 1516 (2009) 